\title{
Numerical calculation of temperature sensing in seawater based on microfibre resonator by intensity-variation scheme
}

\section{H. J. Yang}

J. Wang

S. S. Wang

wangshanshan@ouc.edu.cn

\author{
Department of Physics, College of Information Science and Engineering, Ocean University of China, \\ China \\ Department of Physics, College of Information Science and Engineering, Ocean University of China, \\ China \\ Department of Physics, College of Information Science and Engineering, Ocean University of China, \\ China
}

A seawater temperature sensing and detection method based on microfibre resonator (MR) by intensity-variation scheme is proposed, which has the advantages of high sensitivity and low detection limit. The dependences of sensitivity on probing wavelength, fibre diametre and ring diametre are studied. Results show that probing wavelength influences the sensitivity by the absorption loss predominantly. Larger absorption loss results in lower sensitivity, which is much different with resonant-wavelength-shift scheme. And sensitivity increases with the increasing ring diametre due to the decreasing bending loss and increasing Q-factor. In addition, there may exist an optimal fibre diametre, with which the sensitivity is maximized. By tuning the parameters of system, sensitivity can be tuned from $0.0784 \mathrm{NI} /{ }^{\circ} \mathrm{C}$ to $13.79 \mathrm{NI} /{ }^{\circ} \mathrm{C}$ ( $\mathrm{NI}$ is the abbreviation of normalized intensity). Correspondingly, dynamic range changes from $11.77^{\circ} \mathrm{C}$ to $0.08^{\circ} \mathrm{C}$. Additionally, the dependences of detection limit on wavelength, fibre diametre, and ring diametre are also investigated, which are opposite to that of sensitivity. For different temperatures, the dependences of sensitivity and detection limit at some typical temperatures are studied, which shows that high sensitivity and low detection limit are related to high temperature, and the optimal fibre diametres for high sensitivity and low detection limit are the same at different temperatures. The lowest detection limit is estimated to be $10^{-7 \circ} \mathrm{C}$ level, which is four orders of magnitude smaller than that of the traditional method. Results shown here are beneficial to find the optimal parameters for the temperature sensors, and offer helpful references for assembling micro-photonics device used in seawater sensing and detection.

[DOI: http://dx.doi.org/10.2971/jeos.2014.14047]

Keywords: Microfibres, optical sensors, optical resonators, ring resonators, ocean temperature

\section{INTRODUCTION}

Seawater temperature is one of the most important parameters in oceanographic phenomena. For example, internalwave phenomena happens accompany with seawater temperature fluctuation of about $1.5-3.5^{\circ} \mathrm{C}$ inevitably [1], which indicates that measuring temperature of seawater on line can detect the internal-wave phenomena effectively, and actually it is one of the most widely used methods for internal-wave detecting. Presently, the existing methods for seawater temperature measuring include conductivity-temperature-depth (CTD) system, microwave remote sensing, fibre brag grating and so on [2]-[4]. Among the above methods, CTD is the most popular equipment. However, due to the large size of CTD, the skin temperature of seawater cannot be measured precisely, which limits the application of CTDs in hydrospace detection.

In recent years, sensors based on micro and nanofibres have attracted more and more attentions [5]-[28]. For temperature sensing and detection, sensors based on microfibre resonators (MRs) by resonant-wavelength-shift scheme have been investigated theoretically and demonstrated experimentally [29]-[33], which show sensitivity of $0.017-0.27 \mathrm{~nm} /{ }^{\circ} \mathrm{C}$. Expect for the resonant-wavelength-shift scheme, intensity- variation scheme can potentially provide a higher sensitivity and a simpler setup without the need of a spectrum measurement. But there is little study for the intensity-variation method [34]-[37]. Though for intensity-variation scheme it only functions when the monitoring wavelength lies close to the selected resonance, and this leads to a smaller dynamic range [38]. However, for temperature sensing in seawater the relative small dynamic range is wide enough.

In this paper, based on the MR by the intensity-variation scheme, a method for seawater temperature sensing and detection is proposed, which shows the advantages of high sensitivity and low detection limit. Combining the resonant theory of microfibre ring and empirical formula of seawaters refractive index, we investigate the dependence of transmission intensity on temperature. Then, the dependences of sensitivity on probing wavelength, fibre diametre and ring diametre are studied. Finally, we calculate the detection limit for typical probing wavelength, fibre diametre and ring diametre. Results presented here may offer helpful references for assembling micro-photonics device used in seawater sensing and detection. 


\section{MODELING AND THEORY}

The schematic diagram of the seawater temperature sensing by intensity-variation system based on MR is shown in Figure 1, including optical source, optical spectrum analyzer, power meter, thermometer, temperature-controlled heating platform and $3 \mathrm{~dB}$ coupler. Due that in intensity-variation scheme, temperature change of seawater results in the RI changes of seawater and silica fibre, so we can realize the temperature sensing by monitoring the change of the transmission intensity around the resonant peak. The optical spectrum analyzer helps to find the resonant wavelength conveniently, once the probing wavelength is determined, seawater temperature sensing is mainly completed by monitoring the transmission intensity measured with the power meter.

The transmission intensity can be expressed as follows [39]:

$$
\tau=a^{2} \frac{K+\sigma^{2}-2 \sigma \sqrt{K} \sin \theta}{1+\sigma^{2} K-2 \sigma \sqrt{K} \sin \theta}
$$

where $a$ is the amplitude transmission, $\sigma=a \exp (-\alpha L / 2)$ is the overall amplitude loss per turn, $\alpha$ is the loss, $L$ is the perimeter of the MR, $K$ is the coupling intensity coefficient, $\theta=\beta L$ is the single-pass phase shift of the wave propagation, and $\beta$ is the propagation constant. The propagation constant is obtain from the exact solutions of Helmholtz equations [40, 41],

$$
\begin{gathered}
\left\{\frac{J_{1}^{\prime}(U)}{U J_{1}(U)}+\frac{K_{1}^{\prime}(W)}{W K_{1}(W)}\right\}\left\{\frac{J_{1}^{\prime}(U)}{U J_{1}(U)}+\frac{n_{2}^{2} K_{1}^{\prime}(W)}{n_{1}^{2} W K_{1}(W)}\right\} \\
=\left(\frac{\beta}{k n_{1}}\right)^{2}\left(\frac{V}{U W}\right)^{4},
\end{gathered}
$$

where $U=d / 2\left(k n_{1}^{2}-\beta^{2}\right)^{1 / 2}, W=d / 2\left(\beta^{2}-k n_{2}^{2}\right)^{1 / 2}$, $V=k d / 2\left(n_{1}^{2}-n_{2}^{2}\right)^{1 / 2}, d$ is the diameter of the microfibre, $k$ is the wave number, $n_{1}$ and $n_{2}$ are RIs of silica and seawater, respectively, which is related to the fibre diametre, wavelength and RI of seawater.

The seawater refractive index follows the following empirical formula [42]:

$$
\begin{aligned}
& n(S, T, \lambda)=1.31405 \\
& +\left(1.779 \cdot 10^{-4}-1.05 \cdot 10^{-6} T+1.6 \cdot 10^{-8} T^{2}\right) S \\
& -2.02 \cdot 10^{-6} T^{2}+\frac{15.868+0.01155 S-0.00423 T}{\lambda} \\
& \quad-\frac{4382}{\lambda^{2}}+\frac{1.1455 \cdot 10^{-6}}{\lambda^{3}}
\end{aligned}
$$

where $T$ is the temperature of the seawater, $S$ is the salinity of the seawater, $\lambda$ is the probing wavelength, and $n$ is the refractive index of the seawater. The units of them are ${ }^{\circ} \mathrm{C}, \%$, nm and RIU (Refractive Index Unit), respectively.

\section{NUMERICAL RESULTS}

Based on the above theory, we calculate the resonant spectrum of the MR in seawater firstly, as shown in Figure 2(a). In simulations, we assume that the fibre diametre is $1.5 \mu \mathrm{m}$, ring diametre is $400 \mu \mathrm{m}$, and the salinity of seawater is $35 \%$. Since
Optical Spectrum Analyzer

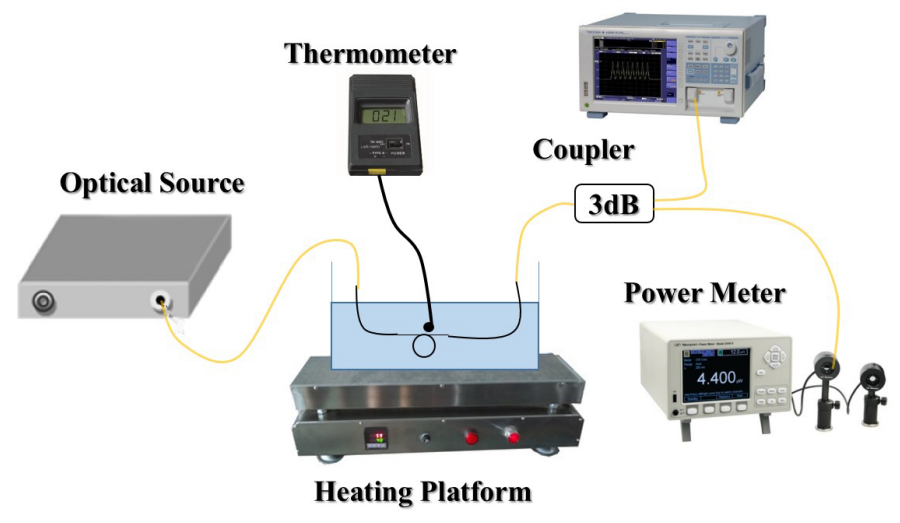

FIG. 1 The schematic diagram of the system based on MR.
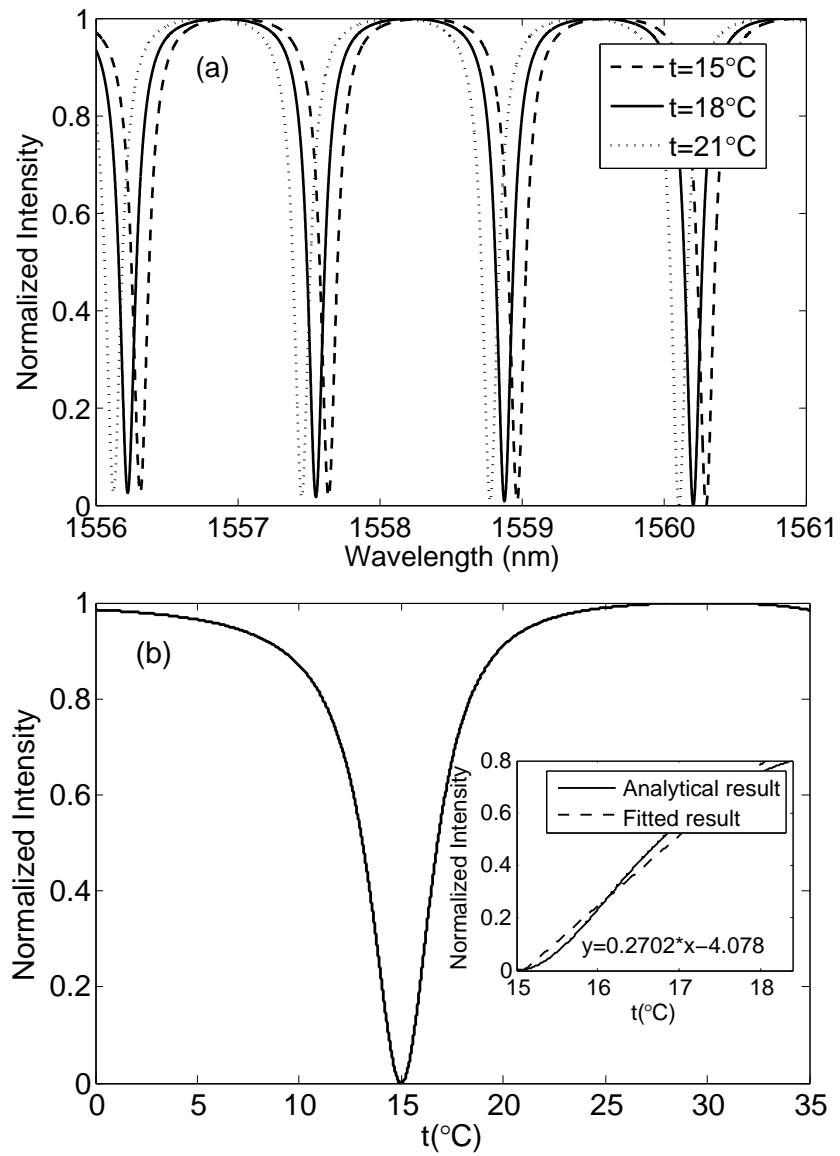

FIG. 2 (a) The resonant spectrum of the MR in the seawater at different temperatures of $15^{\circ} \mathrm{C}, 18^{\circ} \mathrm{C}$ and $21^{\circ} \mathrm{C}$. (b) The normalized intensity as a function of the temperature at $1556.312 \mathrm{~nm}$, the inset indicates the linear relationship of the normalized intensity and the temperature.

the salinity also influences the resonant spectrum, we assume the salinity to be a constant. As shown in Figure 2(a), with the increasing of seawater temperature, the resonant peaks shift due to the RI change of seawater. It can be estimated that the sensitivity of resonant-wavelength-shift scheme is about $0.030 \mathrm{~nm} /{ }^{\circ} \mathrm{C}$. When the resolution of spectral analyzer we used (Ando AQ6370C) is $0.02 \mathrm{~nm}$, the detection limit is estimated to be $0.667^{\circ} \mathrm{C}$, which is much lower than that of CTD's.

For intensity-variation scheme, the sensitivity of the intensityvariation scheme is defined as [38]:

$$
S=\frac{d \tau_{n}}{d t}
$$


where $\tau_{n}$ is the normalized intensity (NI), and $t$ is the temperature of the seawater. To evaluate the sensing sensitivity for intensity-variation scheme, we plot the normalized intensity as a function of the temperature shown in Figure 2(b). In simulations, we choose the wavelength of $1556.312 \mathrm{~nm}$ as the probing wavelength which is the resonant peak. It can be seen from Figure 2(b) that the range of normalized intensity between 0 and 0.8 shows the good linear relationship, so we select this linear range to estimate the sensitivity of intensity-variation scheme, and the slope of the fitted line is considered to be the sensing sensitivity. By this method, the estimated sensitivity shown in Figure 2(b) is about $0.2709 \mathrm{NI} /{ }^{\circ} \mathrm{C}$.

Besides the sensitivity, the detection limit $R$ is another parameter that can evaluate the performance of the system. The detection limit of the system is the smallest change of the temperature that can be detected. The change of temperature effects on the change of the normalization transmission intensity, so the detection limit can be expressed as [38]:

$$
R=\frac{\Delta \tau_{n}}{S}
$$

where $\Delta \tau_{n}$ is the detectable smallest change of the normalization transmission intensity. The detection limit is influenced by the resolution ratio of the instrument, besides the sensitivity. When the resolution of the power meter we used (Newport 918D-IR-OD3) is $0.0004 \%$, the detection limit is $1.48 \cdot 10^{-5 \circ} \mathrm{C}$. Furtherly, the dynamic detection range of the system shown in Figure 2(b) is about $3.38^{\circ} \mathrm{C}$. Considering the internal-wave phenomena happens accompany with the temperature fluctuation of about $1.5-3.5^{\circ} \mathrm{C}$, it is necessary to widen the dynamic detection range of the system by tuning the parameters of system.

In order to expand the dynamic detection range, we decrease the fibre diametre to be $1 \mu \mathrm{m}$ and keep ring diametre $400-\mu \mathrm{m}$. The probing wavelength is chosen to be $1584.376 \mathrm{~nm}$. As is shown in Figure 3(a), the detection range of the intensityvariation scheme extends to be $6.31^{\circ} \mathrm{C}$, which is wide enough for internal-wave sensing. Correspondingly, the sensitivity of the system is about $0.1393 \mathrm{NI} /{ }^{\circ} \mathrm{C}$, which is lower than that of the system in Figure 2(b). This is because that intensityvariation scheme converts the smaller change of the temperature into a large change of the intensity due to the sharp slope near the resonance wavelength. So, if intensity-variation scheme provides a higher sensitivity and a lower detection limit, a narrower dynamic detection range is inevitable. In other words, it is necessary to choose the proper parameters of system according to the real needs. For example, if we want to improve the sensitivity, we can change the system parameters, such as, the probing wavelength to be $1165.08 \mathrm{~nm}$, the fibre diametre to be $1 \mu \mathrm{m}$ and the ring diametre to be $800 \mu \mathrm{m}$. The dependence of the transmission intensity on temperature is shown in Figure 3(b). In this system, the sensitivity is estimated to be $2.556 \mathrm{NI} /{ }^{\circ} \mathrm{C}$. Correspondingly, the dynamic detection range decreases to be $0.38^{\circ} \mathrm{C}$.

To offer more quantitative references for different detection purposes with different sensitivities, we investigate dependences of the sensitivity on the probing wavelength, fibre diametre and ring diametres.
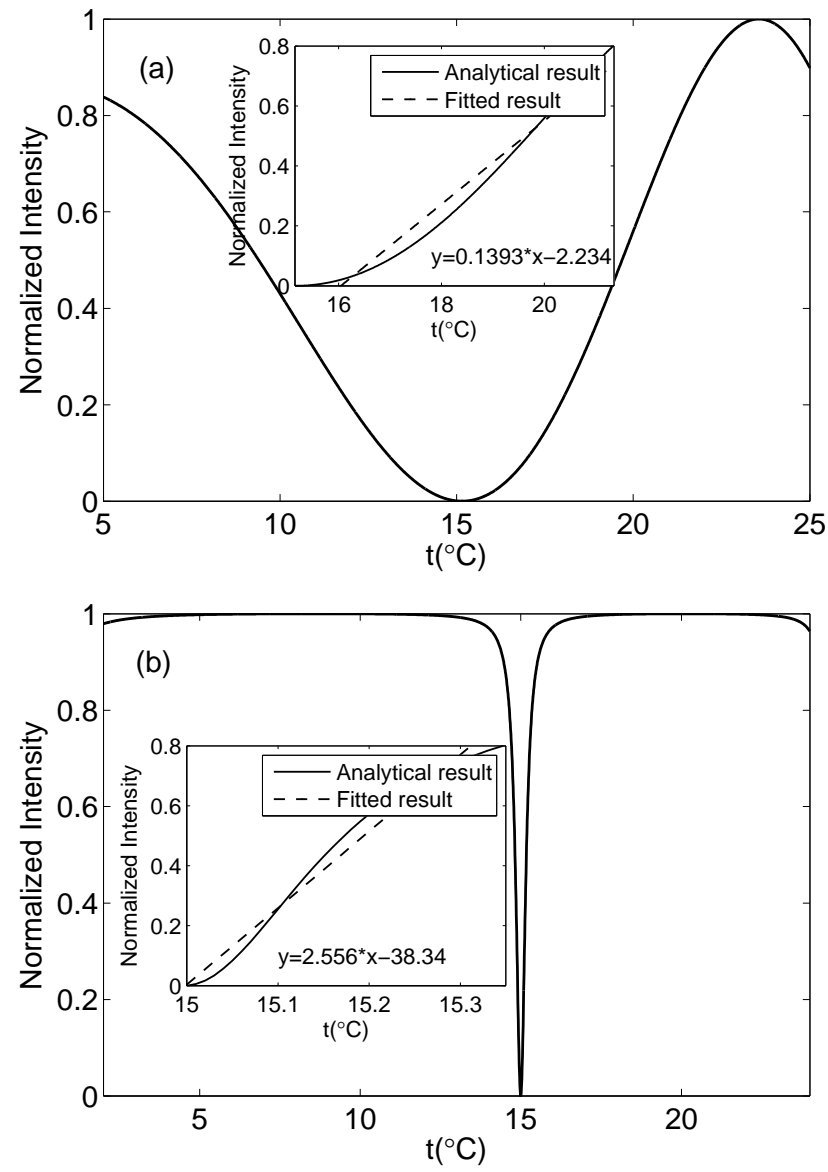

FIG. 3 The normalized intensity as a function of the temperature, the wavelengths are (a) $1584.376 \mathrm{~nm}$ and (b) $1165.08 \mathrm{~nm}$.

\subsection{Dependence of sensitivity on wavelength}

Existing research indicates that in resonant-wavelength-shift scheme sensitivity increases with the increasing probing wavelength $[5,7,21]$, which dues that the microfibres with larger wavelength have higher fractional power transmitted in the seawater, so they are more sensitive to the variation of ambient RI. However, in intensity-variation scheme, sensitivity is not decided by power fraction transmitted in seawater absolutely.

Figure 4 shows the dependence of sensitivity on the probing wavelength. In simulation, the fibre diametre is assumed to be $1 \mu \mathrm{m}$, and the ring diametre is $400 \mu \mathrm{m}$. It can be seen obviously that the dependence of the sensitivity on the probing wavelength is not increasing or decreasing, monotonously, which is much different with resonant-wavelength-shift scheme. It can be explained as follows: in intensity-variation scheme, the sensitivity is a combination of the spectral shift of the resonant mode and the slope of the spectral mode profile and thus the Q-factor. The larger losses, especially, the absorption loss from seawater will result in a lower Q-factor. The losses include guiding loss, bending loss and absorption loss. The evolution and competition of these three losses can be obtained in Reference [21]. The inset in Figure 4 plots the dependence of the absorption coefficient in seawater on the probing wavelength. It can be seen that wavelength with larger absorption coefficient corresponds to the lower sensitivity, which indicates that it is necessary to choose the probing wavelengths with low ab- 




FIG. 4 The dependence of sensitivity on the probing wavelength. Inset: the dependence of the absorption coefficient in seawater on the probing wavelength.

sorption coefficients if we want to increase the sensing sensitivity.

In addition, we calculate the dependences of sensitivity on the probing wavelength for $800-\mu \mathrm{m}$-diametre ring and 1600- $\mu$ m-diametre ring. It can be seen that under the same probing wavelength, larger ring diametre results in higher Q-factor, and we can conclude that the sensitivity can be improved by increasing the ring diametre.

\subsection{Dependence of sensitivity on ring diametre}

To further investigate the dependences of sensitivity on ring diametre, we plot the dependence of the sensitivity on the ring diametre as shown in Figure 5. The fibre diametre used is $1.5 \mu \mathrm{m}$. As we predict, results show that the sensitivity increases with the increasing ring diametre. Specifically, sensitivities increases from $1.053 \mathrm{NI} /{ }^{\circ} \mathrm{C}$ to $4.914 \mathrm{NI} /{ }^{\circ} \mathrm{C}$, $0.601 \mathrm{NI} /{ }^{\circ} \mathrm{C}$ to $1.952 \mathrm{NI} /{ }^{\circ} \mathrm{C}, 0.089 \mathrm{NI} /{ }^{\circ} \mathrm{C}$ to $0.331 \mathrm{NI} /{ }^{\circ} \mathrm{C}$, and $0.078 \mathrm{NI} /{ }^{\circ} \mathrm{C}$ to $0.440 \mathrm{NI} /{ }^{\circ} \mathrm{C}$ under the probing wavelengths of $1064 \mathrm{~nm}, 1310 \mathrm{~nm}, 1450 \mathrm{~nm}$ and $1550 \mathrm{~nm}$. Correspondingly, the dynamic detection ranges are $0.19-0.87^{\circ} \mathrm{C}, 0.47-0.52^{\circ} \mathrm{C}$, $2.67-10.35^{\circ} \mathrm{C}$, and $2.07-11.77^{\circ} \mathrm{C}$, respectively.

\subsection{Dependence of sensitivity on fibre diametre}

Above simulations indicate that sensitivity is dependent on both energy distributed outside microfibre and energy losses induced by guiding loss, bending loss and absorption loss. It is known that larger fibre diametre leads to smaller bending loss and higher sensitivity. However, larger fibre diametre also results in less energy transmitted outside microfibre and reduces the sensitivity. That is to say, there may exist an optimal fibre diametre, with which the sensitivity is maximized. To search the optimal fibre diametre, we calculate the dependence of the sensitivity on the fibre diametre under different probing wavelengths. The ring diametre is $2000 \mu \mathrm{m}$, and the probing wavelengths are close to $1064 \mathrm{~nm}, 1310 \mathrm{~nm}$ and $1550 \mathrm{~nm}$, respectively. As shown in Figure 6, one maximum of sensitivity can be found for systems with probing wavelengths of $1064 \mathrm{~nm}$ and $1310 \mathrm{~nm}$. Specifically, the optimal diametres are $750 \mathrm{~nm}$ and $950 \mathrm{~nm}$, and the maximum sensitiv-

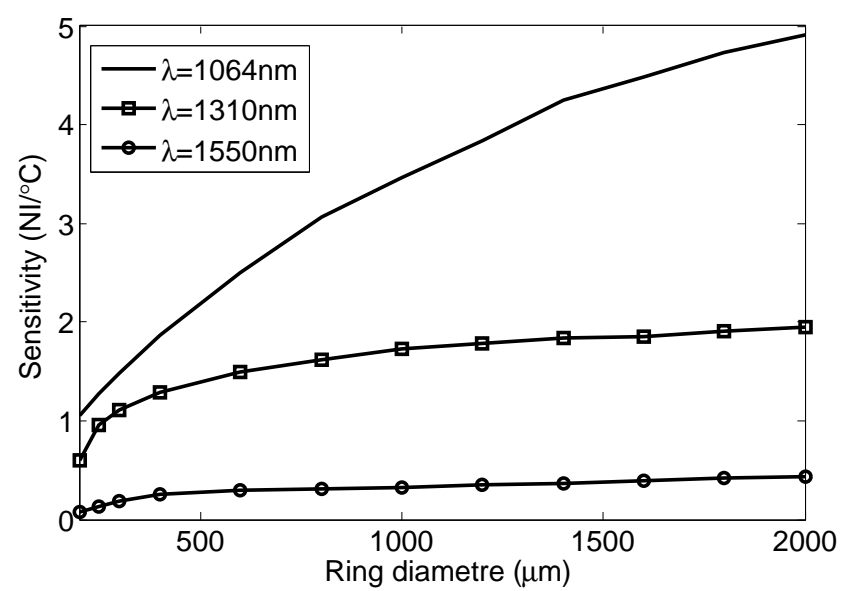

FIC. 5 The dependence of sensitivity on the ring diametre.

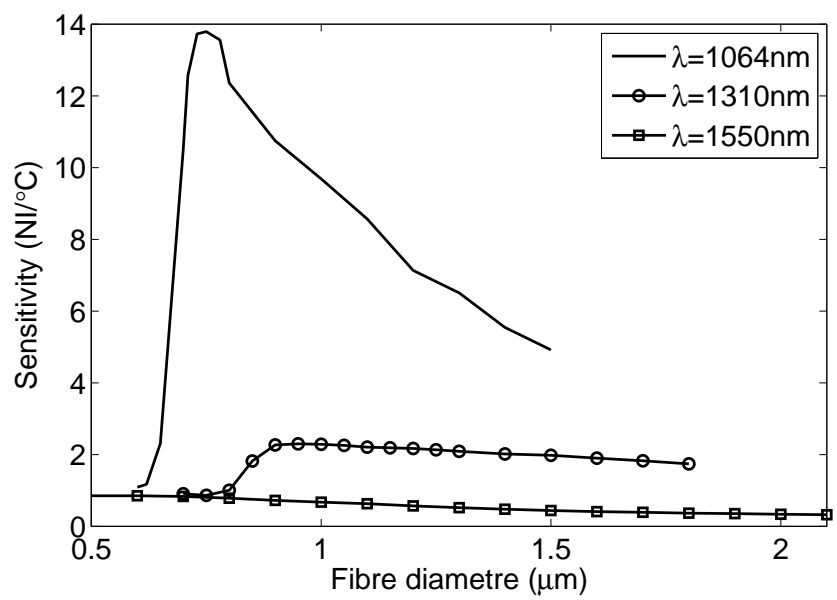

FIG. 6 The dependence of sensitivity on the ring diametre.

ities are $13.78 \mathrm{NI} /{ }^{\circ} \mathrm{C}$ and $2.299 \mathrm{NI} /{ }^{\circ} \mathrm{C}$, respectively. The relationship shown in Figure 6 may help us to maximize the sensitivity by choosing the fibre diametre.

\subsection{Detection limit}

The dependences of detection limit on wavelength, ring diametre, and fibre diametre are shown in Figure 7. As is known in Eq. (5), the detection limit has opposite dependences on wavelength, ring diametre, and fibre diametre compared to sensitivity. The low detection limit is related to short wavelength, large ring diameter. Additionally, there may exist an optimal fibre diametre, with which the detection limit is minimum. If the resolution ratio of the power meter (Newport 918D-IR-OD3) is $0.0004 \%$, the probing wavelength is $1064 \mathrm{~nm}$, the fibre diametre is $0.75 \mu \mathrm{m}$, and the MR diametre is $2000 \mu \mathrm{m}$, the sensitivity can reach to $13.78 \mathrm{NI} /{ }^{\circ} \mathrm{C}$, and the calculated detection limit is $2.903 \cdot 10^{-7 \circ} \mathrm{C}$, which is lower than that of CTD system and shows great potential in fine measuring of temperature in seawater. To find the optimal performance of the sensor at different temperature, the dependences of sensitivity and detection limit on fibre diametre at some typical temperatures are plotted in Figure 8. The probing wavelength is assumed to be $1310 \mathrm{~nm}$. It can be seen that high sensitivity and low detection limit are related to high temperature, which is due that the change in the RI of seawater decreases the loss at high temperature and thus increases the Q-factor [16]. Additionally, the optimal fibre diametres for high sensitivity and low detection limit are the same at different temperatures, 

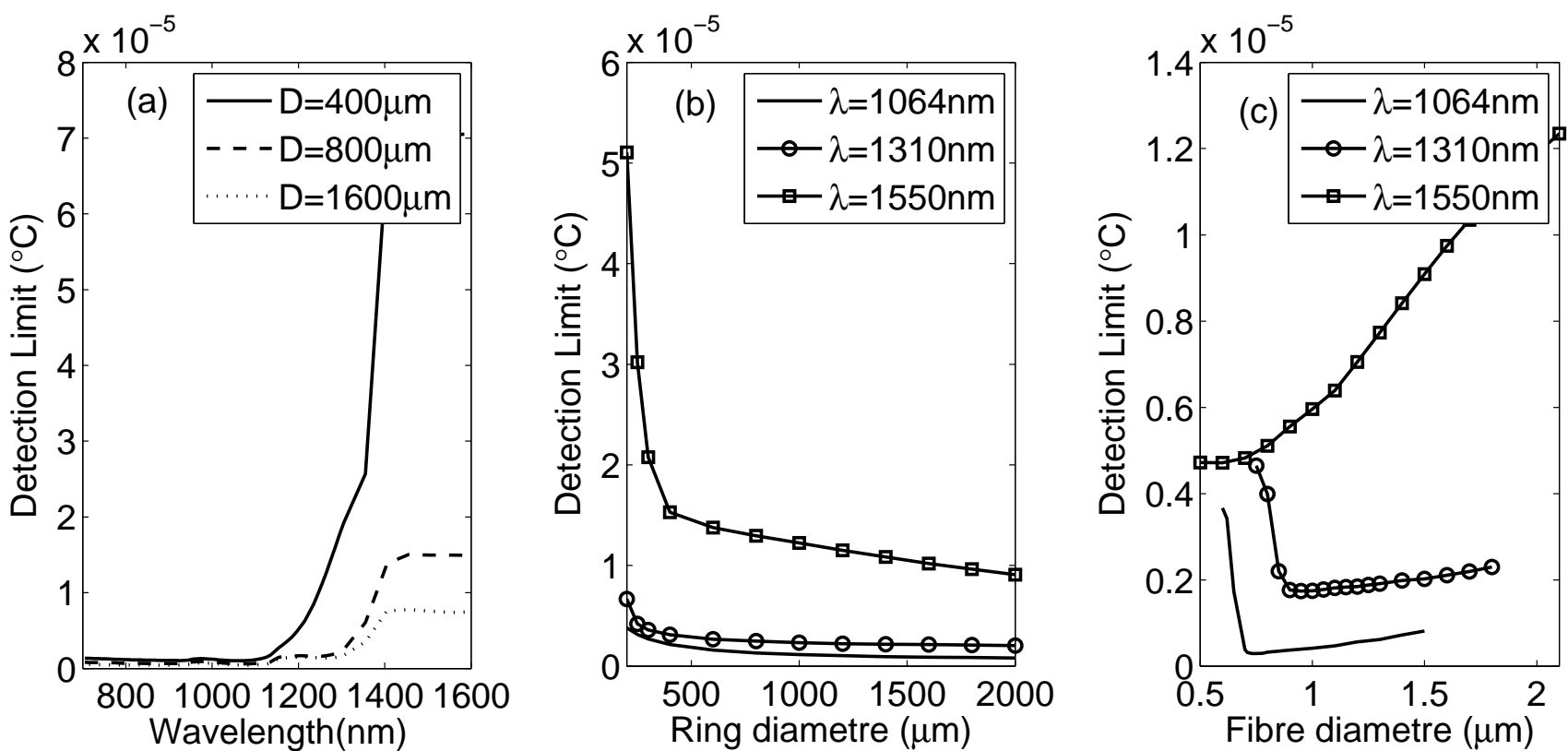

FIG. 7 The dependences of detection limit on (a) wavelength, (b) ring diametre, and (c) fiber diametre.
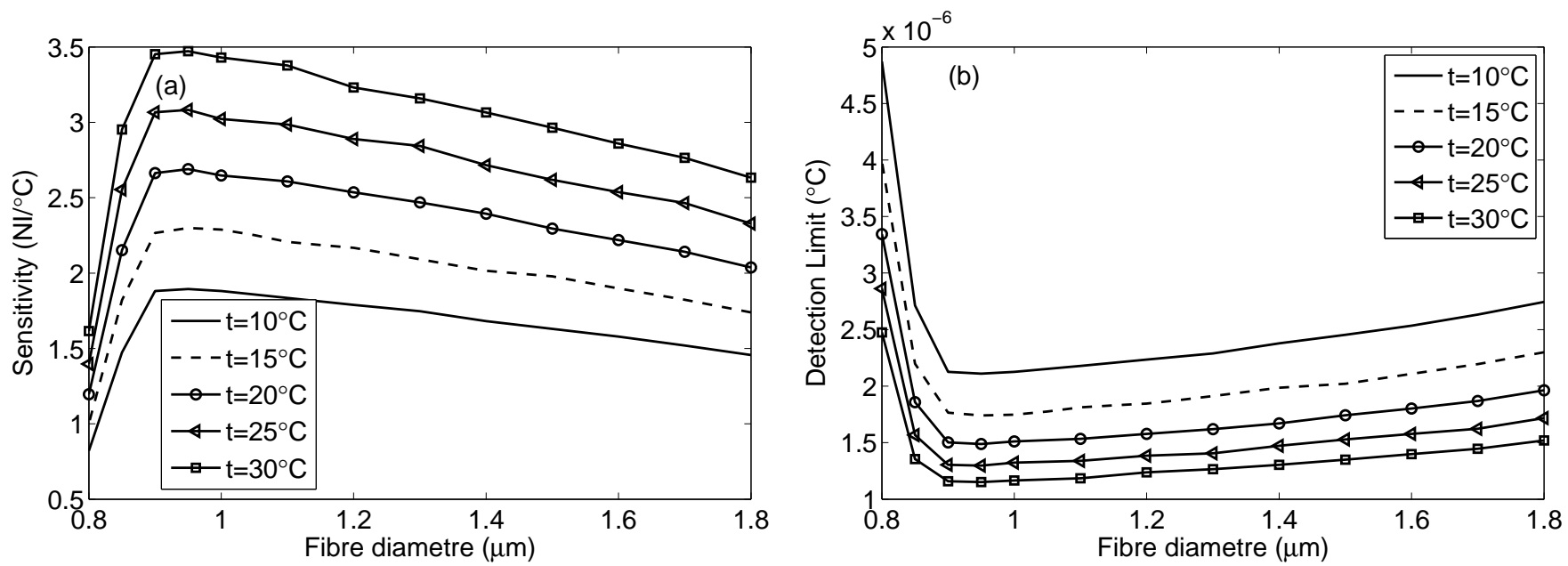

FIG. 8 The dependences of (a) sensitivity and (b) detection limit on fibre diametre at some typical temperatures.

which indicates that the optimal fibre diametre for MR with the same ring diametre is mainly determined by the probing wavelength.

\section{CONCLUSION}

In conclusion, we have numerically studied temperature sensing in seawater based on microfibre resonator by intensityvariation scheme. Dependences of the sensitivity on probing wavelength, ring diametre and fibre diametre are investigated. Results show that the sensitivity of temperature sensing is determined by both the fraction of power outside the fibre and the losses induced by bending and absorption. The probing wavelength influences the sensitivity mostly by the absorption loss. Larger absorption loss results in lower sensitivity, which is much different with resonant-wavelengthshift scheme. And the sensitivity increases with the increasing ring diametre due to the decreasing bending loss and increasing Q-factor. In addition, there may exist an optimal fibre diametre, with which the sensitivity is maximized. By tuning the above parameters of system, sensitivity can be tuned from $0.0784 \mathrm{NI} /{ }^{\circ} \mathrm{C}$ to $13.79 \mathrm{NI} /{ }^{\circ} \mathrm{C}$. Correspondingly, dynamic range changes from $11.77^{\circ} \mathrm{C}$ to $0.08^{\circ} \mathrm{C}$. Additionally, the dependences of detection limit on wavelength, fibre diametre, and ring diametre are also investigated, which are opposite to that of sensitivity. For different temperatures, the dependences of sensitivity and detection limit at some typical temperatures are studied, which shows that high sensitivity and low detection limit are related to high temperature and the optimal fibre diametres for high sensitivity and low detection limit are the same at different temperatures. The calculated detection limit is about $10^{-7 \circ} \mathrm{C}$, which is four orders of magnitude smaller than that of the traditional method. Results shown here are beneficial to find the optimal parameters for the temperature sensors, and offer helpful references for assembling micro-photonics device used in seawater sensing and detection.

\section{ACKNOWLEDGEMENTS}

The authors would like to thank financial supports from the Natural Science Foundations of China (No. 61171161 and 61405181) and the Fundamental Research Project of Qingdao (No. 12-1-4-1-(26)-jch). 


\section{References}

[1] X. H. Fang, F. M. Boland, and G. R. Cresswell, "Further observations of high-frequency current variations on the continental shelf near Sydney, New South Wales," Aust. J. Mar. Fresh. Res. 35, 611-618 (1984).

[2] R. Millard, J. Toole, and M. Swartz, "A fast responding temperature measurement system for CTD applications," Ocean Eng. 7, 413-427 (1980).

[3] C. T. Swift, "Passive microwave remote sensing of the ocean - A review," Bound-Lay. Meteorol. 18, 25-54 (1980).

[4] D. A. Pereira, 0. Frazao, and J. L. Santos, "Fibre Bragg grating sensing system for simultaneous measurement of salinity and temperature," Opt. Eng. 43, 299-304 (2004).

[5] F. Xu, P. Horak, and G. Brambilla, “Optical microfiber coil resonator refractometric sensor," Opt. Express 15, 7888-7893 (2007).

[6] F. Xu, and G. Brambilla, "Demonstration of a refractometric sensor based on optical microfiber coil resonator," Appl. Phys. Lett. 92, 101126 (2008).

[7] F. Xu, V. Pruneri, V. Finazzi, and G. Brambilla, "An embedded optical nanowire loop resonator refractometric sensor," Opt. Express $16,1062-1067$ (2008).

[8] X. Guo, and L. M. Tong, "Supported microfiber loops for optical sensing," Opt. Express 16, 14429-14434 (2008).

[9] J. Villatoro, M. P. Kreuzer, R. Jha, V. P. Minkovich, V. Finazzi, G. Badenes, and V. Pruneri, "Photonic crystal fiber interferometer for chemical vapor detection with high sensitivity," Opt. Express $17,1447-1453$ (2009).

[10] G. Coviello, V. Finazzi, J. Villatoro, and V. Pruneri, "Thermally stabilized PCF-based sensor for temperature measurements up to $1000^{\circ} \mathrm{C}$," Opt. Express 17, 21551-21559 (2009).

[11] R. Jha, J. Villatoro, G. Badenes, and V. Pruneri, "Refractometry based on a photonic crystal fiber interferometer," Opt. Lett. 34, 617-619 (2009).

[12] J. L. Kou, J. Feng, L. Ye, F. Xu, and Y. Q. Lu, “Miniaturized fiber taper reflective interferometer for high temperature measurement," 0pt. Express 18, 14245-14250 (2010).

[13] N. Lou, R. Jha, J. L Domínguez-Juárez, V. Finazzi, J. Villatoro, G. Badenes, and V. Pruneri, "Embedded optical micro/nano-fiber for stable devices," Opt. Lett. 35, 571-573 (2010).

[14] G. A. Cárdenas-Sevilla, V. Finazzi, J. Villatoro, and V. Pruneri, "Photonic crystal fiber sensor array based on modes overlapping," Opt. Express 19, 7596-7602 (2011).

[15] C. R. Liao, D. M. Wang, X. Y. He, and M. W. Yang, "Twisted optical microfibers for refractive index sensing," IEEE Photonic. Technol. Lett. 23, 848-850 (2011).

[16] S. W. Harun, K. S. Lim, S. S. A. Damanhur, and H. Ahmad, "Microfiber loop resonator based temperature sensor," J. Europ. Opt. Soc. Rap. Public. 6, 11026 (2011).

[17] K. S. Lim, I. Aryanfar, W. Y. Chong, Y. K. Cheong, S. W. Harun, and H. Ahmad, "Integrated microfibre device for refractive index and temperature sensing," Sensors 12, 11782-11789 (2012).

[18] L. P. Sun, J. Li, Y. Z. Tan, X. Shen, X. D. Xie, S. Gao, and B. O. Guan, "Miniature highly-birefringent microfiber loop with extremely-high refractive index sensitivity," Opt. Express 20, 10180-10185 (2012).

[19] J. H. Wo, G. H. Wang, Y. Cui, Q. Z. Sun, R. B. Liang, P. P. Shum, and D. M. Liu, "Refractive index sensor using microfiber-based Mach-Zehnder interferometer," Opt. Lett. 37, 67-69 (2012).
[20] W. B. Ji, H. H. Liu, S. C. Tjin, K. K. Chow, and A. Lim, "Ultrahigh sensitivity refractive index sensor based on optical microfiber," IEEE Photonic. Technol. Lett. 24, 1872-1874 (2012).

[21] S. S. Wang, J. Wang, G. Li, and L. Tong, "Modeling optical microfiber loops for seawater sensing," Appl. Opt. 51, 3017-3023 (2012).

[22] G. Y. Chen, M. Ding, T. P. Newson, and G. Brambilla, "A review of microfiber and nanofiber based optical sensors," Open Optic. J. 7, 32-57 (2013).

[23] L. Bo, P. F. Wang, Y. Semenova, and G. Farrell, "High sensitivity fiber refractometer based on an optical microfiber coupler," IEEE Photonic. Technol. Lett. 25, 228-230 (2013).

[24] G. Y. Chen, G. Brambilla, and T. P. Newson, "Inspection of electrical wires for insulation faults and current surges using sliding temperature sensor based on optical Microfibre coil resonator," Electron. Lett. 49, 46-47 (2013).

[25] M. Z. Muhammad, A. A. Jasim, H. Ahmad, H. Arof, and S. W. Harun, "Non-adiabatic silica microfiber for strain and temperature sensors," Sensor. Actuat. A-Phys. 192, 130-132 (2013).

[26] Y. S. Chiam, K. S. Lim, S. W. Harun, S. N. Gan, and S. W. Phang, "Conducting polymer coated optical microfiber sensor for alcohol detection," Sensor. Actuat. A-Phys. 205, 58-62 (2014).

[27] Y. Wu, B. C. Yao, Y. Cheng, Y. J. Rao, Y. Cong, W. L. Zhang, Z. G. Wang, et al., "Hybrid graphene-microfiber waveguide for chemical gas sensing," IEEE J. Sel. Top. Quant. 20, 4400206 (2014).

[28] J. Y. Lou , Y. P. Wang, and L. M. Tong, "Microfiber optical sensors: A review," Sensors 14, 5823-5844 (2014).

[29] M. Sumetsky, Y. Dulashko, J. M. Fini, A. Hale, and D. J. DiGiovanni, "The microfibre loop resonator: theory, experiment, and application," J. Lightwave Technol. 24, 242-250 (2006).

[30] X. Zeng, Y. Wu, C. L. Hou, J. Bai, and G. G.Yang, "A temperature sensor based on optical microfibre knot resonator," Opt. Commun. 282, 3817-3819 (2009).

[31] Y. Wu, Y. J. Rao, Y. H. Chen, and Y. Cong, “Miniature fiber-optic temperature sensors based on silica/polymer microfibre knot resonators," Opt. Express 17, 18142-18147 (2009).

[32] Y. Chen, Y. Ming, W. Guo, F. Xu, and Y. Q. Lu, "Temperature characteristics of microfiber coil resonators embedded in teflon," in Proceedings to the Communications and Photonics Conference and Exhibition, ACP. Asia, 1-6 (Shanghai Jiaotong University, Shanghai, 2011).

[33] Y. Wu, L. Jia, T. H. Zhang, Y. J. Rao, and Y. Cong, "Microscopic multi-point temperature sensing based on microfiber double-knot resonators," Opt. Commun. 285, 2218-2222 (2012).

[34] C. Y. Chao, and L. J. Guo, "Biochemical sensors based on polymer microrings with sharp asymmetrical resonance," Appl. Phys. Lett. 83, 1527-1529 (2003).

[35] C. Y. Chao, W. Fung, and L. J. Cuo, "Polymer microring resonators for biochemical sensing applications," IEEE J. Sel. Top. Quant. 12, 134-142 (2006).

[36] H. X. Yi, D. S. Citrin, and Z. P. Zhou, "Highly sensitive athermal optical microring sensor based on intensity detection," IEEE J. Quantum Elect. 47, 354-358 (2011).

[37] C. Qiu, T. Hu, P. Yu, A. Shen, F. Wang, X. Q. Jiang, and J. Y. Yang, "A temperature sensor based on silicon eye-like microring with sharp asymmetric fano resonance," in Proceedings to the 2012 IEEE gth International Conference on Group IV Photonics (GFP), 123-125 (IEEE, San Diego, 2012). 
[38] C. Y. Chao, and L. J. Guo, "Design and optimization of microring resonators in biochemical sensing applications," J. Lightwave Technol. 24, 1395-1402 (2006).

[39] 0. Schwelb, "Transmission, group delay, and dispersion in singlering optical resonators and add/drop filters-a tutorial overview," J. Lightwave Technol. 22, 1380-1394 (2004).

[40] A. W. Snyder, and J. D. Love, Optical waveguide theory (Chapman and Hall, New York, 1991).
[41] L. M. Tong, J. Y. Lou, and E. Mazur, "Single-mode guiding properties of subwavelength-diameter silica and silicon wire waveguides," Opt. Express 12, 1025-1035 (2004).

[42] X. H. Quan, and E. S. Fry, "Empirical equation for the index of refraction of seawater," Appl. Opt. 34, 3477-3480 (1995). 